\title{
Pas de numérisation sans interfaces fonctionnelles
}

\section{Alexander Zimmer}

Dr méd., membre du Comité central et responsable du département Numérisation/eHealth

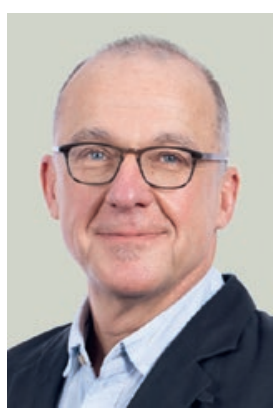

Avez-vous remarqué que les fabricants de smartphones, de tablettes et d'écrans d'ordinateur tendent à faire rétrécir toujours plus la bordure de l'écran? Ceci n'est pas sans fâcher les adeptes du post-it. Il n'y a plus guère que le fabricant à la pomme qui ménage encore un généreux espace au bas de l'écran où coller nos petites notes au stylo. La transformation numérique se traduit différemment selon le domaine et l'acteur concernés, un constat qui se vérifie au niveau des autorités fédérales.

Les difficultés rencontrées au début dans la gestion de la pandémie ont conduit plusieurs parlementaires à déposer une motion appelant à renforcer la numérisation dans le domaine de la santé. Notre pays est en retard dans ce domaine, comme le montre le classement Digital Health Index de la fondation Bertelsmann, où la Suisse reste $14^{\mathrm{e}}$ sur 17. L'OFSP a réagi avec célérité et renforcé sa stratégie cybersanté en créant une division Transformation numérique. Une des conclusions de la sixième conférence nationale Santé2020, en 2019, a été que la numérisation crée les bases nécessaires à un système de santé plus performant et plus sûr, notamment en mettant en réseau les professionnels et les institutions du secteur de la santé.

Les choses avancent. On trouve néanmoins sur la page de l'OFSP dédiée aux maladies infectieuses à déclaration obligatoire, alors que nous sommes en deuxième année de pandémie, l'injonction faite aux laboratoires

\section{Pour une transformation numérique qui} n'entraîne pas une surcharge administrative massive, il faut des interfaces fonctionnelles.

de communiquer les résultats d'analyses via un "formulaire de déclaration» à envoyer par fax ou par courrier postal. La même page mentionne quand même que l'OFSP prévoit de mettre en place un système électronique.

L'avantage propre à la numérisation serait pourtant que les données, une fois saisies, soient utilisées plusieurs fois, ce qui éviterait aux médecins de fastidieuses saisies à double comme l'exigent les actuels formulaires électroniques.
La FMH, par son implication dans la future communauté DEP AS Swiss, s'engage pour que les médecins puissent réutiliser les données saisies au quotidien dans le système primaire de leur cabinet, par exemple pour la transmission des données relatives aux vaccinations à l'OFSP, sans avoir à saisir une nouvelle fois les mêmes données.

\section{Nous mettons tout en œuvre pour que nos} membres puissent réutiliser les données saisies dans le cadre de leur travail quotidien.

Hélas, ce n'est pas ce que semblent souhaiter tous les cantons, certains voulant conserver leur propre solution pour la saisie des données vaccinales. Honni soit qui mal y pense, mais est-il judicieux d'opposer le transfert direct de données entre le système primaire des cabinets et l'OFSP, pour que les médecins s'acquittent de leur obligation de déclarer, à la mise en place de registres de vaccination à l'échelon de l'administration fédérale ou des cantons?

Le principe voulant que les données soient saisies une fois pour pouvoir être utilisées plusieurs fois n'a pas uniquement pour avantage d'offrir aux patients un meilleur contrôle des informations les concernant. Alors que la numérisation progresse, préserver et encourager l'autonomie des médecins pour ce qui est de la gestion des données constitue un objectif prioritaire pour la FMH. Dans le domaine des tarifs, les médecins bénéficient d'ores et déjà des données collectées par le biais de Trustcenter et de NewIndex.

A première vue, les post-it évoqués plus haut ont certains avantages: ils sont rapides à mettre en œuvre et donc efficaces. En même temps, ils sont révélateurs du fait que la révolution numérique n'a pas encore abouti. Au plus tard lorsqu'il s'agira d'échanger ou de réutiliser les informations notées sur les post-it, il faudra une nouvelle fois les saisir et les transmettre, comme dans une comptabilité en partie double. Or, ce doublon n'est pas nécessaire en soi, et c'est pourquoi la FMH s'engage résolument pour que l'on résolve le problème des interfaces et que l'on puisse échanger des données médicales de manière efficace et structurée. 